\title{
Una Mirada a la Teoría de Representaciones de Grupos Finitos
}

\author{
Luis Yair Meza Pérez \\ Carlos Pompeyo-Gutiérrez \\ Universidad Juárez Autónoma de Tabasco \\ e-mail: matematico_meza@hotmail.com \\ carlos.pompeyo@ujat.mx
}

\begin{abstract}
Resumen
La teoría de representaciones de grupos finitos estudia, grosso modo, simetrías en espacios lineales y uno de sus objetivos condensar el máximo de información de un grupo con un mínimo de datos. Esta poderosa herramienta tiene múltiples alcances, tanto dentro de las mismas matemáticas (teoría de números, geometría algebraica, etc.) como en otras ramas de la ciencia fuera de ellas, por ejemplo física y química. En este trabajo no se pretende mostrar resultados nuevos, sino más bien dar una breve reseña histórica de la teoría de representaciones, comentar la relación de ésta con la teoría de caracteres y presentar ejemplos ilustrativos que hagan más comprensibles las ideas al lector.
\end{abstract}

\section{Antecedentes históricos}

La teoría de representaciones de grupos finitos nace en 1896, resultado del trabajo del matemático alemán Ferdinand Georg Frobenius en un intento más o menos deliberado de generalizar la teoría de caracteres de grupos abelianos finitos. Ésta le serviría como herramienta para la solución de un problema planteado por su colega y compatriota Richard Dedekind, a través de una carta de éste último al primero. En ella le hace la siguiente observación: tomar la tabla de multiplicar de un grupo finito $G$ y transformarla en una matriz $X_{G}$ reemplazando cada entrada $g$ de dicha tabla por una variable $x_{g}$. De esta manera, el determinante de $X_{G}$ se puede escribir como un producto de polinomios irreducibles en las variables $\left\{x_{g}\right\}_{g \in G}$, cada uno de los cuales tiene multiplicidad igual a su grado [3, pág. 4]. Dedekind únicamente pudo verificar este hecho para algunos casos particulares de grupos abelianos, pero no lo pudo generalizar para grupos finitos no conmutativos, por lo que recurre a Frobenius. Éste le responde a Dedekind el 12 de abril de 1896, describiéndole sus ideas sobre la factorización de un cierto polinomio homogéneo asociado a un grupo finito conocido como grupo determinante [6, pág. 361]. El intercambio de correspondencia continuó los días 17 y 26 de abril de 1896, donde para el día 30 de ese mismo mes y año Frobenius tenía ya los fundamentos de la teoría de caracteres de grupos finitos. Tomaría algún tiempo para que el desarrollo de su idea de representación de un grupo finito estuviese completa, pero la correspondencia Frobenius-Dedekind en aquel abril de 1896, hace ya 122 años, es considerada como el suceso que marcó el nacimiento de la teoría de representaciones de grupos finitos. 


\section{Trascendencia}

La comprensión de las propiedades de un grupo o un conjunto de ellos puede ser complicado, o por lo menos tedioso, dependiendo de la forma en que éstos sean descritos. Así, resulta conveniente contar con métodos que permitan asociarle a grupos abstractos, objetos matemáticos concretos con los cuales el estudio de aquellas propiedades de interés en los primeros sea más sencillo. En este sentido, una técnica que ofrece tales ventajas es la teoría de representaciones, ya que permite transformar (de alguna manera), problemas abstractos de teoría de grupos en problemas equivalentes (pero relativamente más simples y manejables) de álgebra lineal. De este modo es posible representar un grupo $G$ como un grupo de matrices invertibles, con lo que las herramientas del álgebra matricial serán de gran utilidad a la hora de obtener la información deseada sobre el grupo que se estudia.

Dentro de la matemática misma, la teoría de caracteres hace posible la prueba de dos célebres resultados, puramente teóricos, acerca de las propiedades de grupos abstractos:

Teorema (Burnside, 1904). Si p y q son primos distintos, entonces todo grupo de orden $p^{\alpha} q^{\beta}$ es soluble, para cualesquiera enteros no negativos $\alpha$ y $\beta$.

Teorema (Frobenius, 1901). Sea G un grupo finito que actúa transitivamente sobre un conjunto $X$ de cardinalidad $n$. Si todas las permutaciones ${ }^{1}$ de $G$, salvo la identidad, tienen a lo más un punto fijo, entonces el conjunto de elementos de $G$ sin puntos fijos, junto con la identidad, es un subgrupo normal de $G$ de orden $n$.

Este último se puede reformular, en abstracto, como sigue:

Teorema (Frobenius). Si $H$ es un subgrupo de un grupo finito $G$ con la propiedad de que $\sigma H \sigma^{-1} \cap H=\{e\}$, para todo $\sigma \in G-H$, entonces $G$ tiene un subgrupo normal $K$ tal que $G=K H$ y $H \cap K=\{e\}$.

Para que el lector pueda hacerse una idea del poder de la teoría de representaciones: a diferencia del teorema $p^{\alpha} q^{\beta}$ de Burnside, para el que tomó siete décadas hallar una demostración sin usar caracteres (H. Bender presentó su prueba en 1972), todas las demostraciones conocidas hasta ahora del teorema de Frobenius utilizan la teoría de caracteres de una forma u otra [8, pág. 225].

No obstante, el alcance de la teoría de representaciones va más allá de las matemáticas. En [2, III, págs. 115-179], el autor dedica tres extensos capítulos de su trabajo para presentar varias aplicaciones de esta teoría en la física, concretamente en la mecánica cuántica: Átomos (Capítulo 7), Moléculas (Capítulo 8) y Sólidos (Capítulo 9), centrándose en el estudio de las simetrías en estos objetos físicos. La química tampoco se queda atrás: en la última sección de [7], los autores presentan algunos ejemplos de aplicaciones de la teoría de representaciones en espectroscopía infrarroja y de Raman, donde además construyen la tabla de caracteres de cada uno de los grupos de simetrías que estudian.

\footnotetext{
${ }^{1}$ Por el Teorema de Cayley, todo grupo $G$ es isomorfo a un subgrupo de un grupo de permutaciones. La acción de un grupo $G$ en un conjunto $X$ induce un homomorfismo de grupos $\varphi: G \rightarrow S_{X}$. Como $|X|=n$, se sigue que $S_{X}=S_{n}$ y en consecuencia $\varphi(G)<S_{n}$. Luego, si ker $\varphi=\{e\}$, entonces se tendrá una imagen fiel de $G$ en $S_{n}$ y por lo tanto será posible hacer la identificación de los elementos de $G$ con permutaciones.
} 


\section{Teoría de Representaciones de Grupos Finitos}

Conviene precisar que en adelante, salvo que se diga otra cosa, por grupo entenderemos grupo finito y por espacio vectorial entenderemos $\mathbb{C}$-espacio vectorial de dimensión finita.

\subsection{Representaciones de Grupos}

Recordemos que si $V$ es un $\mathbb{C}$-espacio vectorial de dimensión $n$, el conjunto End $(V)$ de las transformaciones lineales de $V$ en sí mismo (operadores lineales) es un $\mathbb{C}$-espacio vectorial de dimensión $n^{2}$. Las unidades de End $(V)$ — esto es, los operadores lineales que son invertiblesforman el grupo general lineal GL $(V)$. Más aún, fijando una base de $V$, a cada operador lineal corresponde una única matriz de $n \times n$ con entradas complejas, por lo que End $(V)$ es isomorfo (como anillo y como espacio vectorial) a Mat ${ }_{n \times n}(\mathbb{C})$. En este caso, el grupo GL $(V)$ se denotará por $\mathrm{GL}(n, \mathbb{C})$ y es el grupo multiplicativo de las matrices invertibles de $n \times n$ con entradas complejas.

Definición 3.1. a) Una representación de un grupo $G$ sobre un espacio vectorial $V$ es un homomorfismo $\rho: G \longrightarrow G L(V)$ de $G$ al grupo de automorfismos de $V$.

b) Un morfismo entre dos representaciones $(V, \rho)$ y $(W, \tilde{\rho})$ de un grupo $G$ es una transformación lineal $\varphi: V \longrightarrow W$ tal que para todo $g \in G, \varphi \circ \rho(g)=\tilde{\rho}(g) \circ \varphi$. Esto es, el diagrama

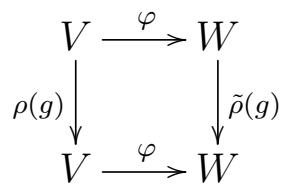

es conmutativo. Si además $\varphi$ es un isomorfismo, diremos que $V$ y $W$ son representaciones isomorfas o equivalentes.

c) El grado de una representación es la dimensión de $V$.

Trabajar en abstracto con la definición anterior puede resultar un tanto difícil. Sin embargo, existe una definición alternativa de representación que puede hacer más accesible la idea y facilitar, en algunos casos, los cálculos.

Definición 3.2 (Alternativa). 1 ) Una representación $\rho$ es un homomorfismo de grupos $\rho: G \longrightarrow G L(n, \mathbb{C})$ que asocia a cada elemento $g$ del grupo $G$ (abstracto) una matriz (concreta) invertible $\rho(g)$.

2) Un morfismo entre dos representaciones $(V, \rho)$ y $(W, \tilde{\rho})$ de un grupo $G$, es una matriz $P \in \operatorname{Mat}_{n \times n}(\mathbb{C})$ tal que $P \rho(g)=\tilde{\rho}(g) P$. Si $P$ es invertible, entonces $\rho(g)=P^{-1} \tilde{\rho}(g) P$ en cuyo caso diremos que $(V, \rho)$ y $(W, \tilde{\rho})$ son isomorfas o equivalentes.

3) A V se le denomina espacio de la representación. 
Observación. El uso de las notaciones $(V, \rho)$ o $V$ para denotar una misma representación $\rho$ de $G$ tiene lugar cuando se desea enfatizar el espacio $V$ o el homomorfismo $\rho$ se sobreentiende y no hay riesgo de confusión, respectivamente.

Ahora se presentarán una serie de ejemplos ilustrativos con la finalidad de hacer más comprensibles al lector las definiciones anteriores y las ideas subyacentes, al mismo tiempo de irse familiarizando poco a poco con la teoría.

Ejemplo 3.3. Si $S_{n}$ es el grupo simétrico, la función signo sgn : $S_{n} \longrightarrow\{+1,-1\} \subset \mathbb{C}^{*}$, donde $\mathbb{C}^{*}$ es el grupo de unidades de $\mathbb{C}$, es una representación de grado uno en virtud de que $\mathbb{C}^{*}$ es isomorfo a $G L(1, \mathbb{C})$.

Ejemplo 3.4. Sea $G$ un grupo arbitrario y definamos $\rho: G \longrightarrow \mathbb{C}^{*} \operatorname{como} \rho(g):=1$ para todo $g \in G$. Esta es una representación de grado uno denominada representación trivial o principal de $G$.

Ejemplo 3.5. Sea $G$ un grupo de orden $n$. Una representación de grado uno es un homomorfismo $\rho: G \longrightarrow \mathbb{C}^{*}$. Como $|G|=n$, entonces $g^{n}=$ e para todo $g \in G$ y dado que $\rho$ es un homomorfismo, entonces $1=\rho\left(g^{n}\right)=\rho(g)^{n}$. Por consiguiente, el número complejo $\rho(g) \in \mathbb{C}^{*}$ es una raíz $n$-ésima de la unidad. En particular, $|\rho(g)|=1$.

Ejemplo 3.6 (La representación regular). Sea $G$ un grupo finito de orden $n$ y sea $V$ un $\mathbb{C}$ espacio vectorial de dimensión $n$ con base $\mathcal{B}=\left\{v_{g}\right\}_{g \in G}$ indizada por los elementos del grupo. Para cada $\sigma \in G$ definimos la función $\rho(\sigma): \mathcal{B} \longrightarrow \mathcal{B}$ como $\rho(\sigma)\left(v_{g}\right)=v_{\sigma g}$ y observemos que $\rho(\sigma)$ es una biyección. Entonces, la función $\rho(\sigma)$ puede extenderse linealmente a un isomorfismo de espacios vectoriales $\rho(\sigma): V \longrightarrow V$. La función

$$
\rho: G \longrightarrow G L(V) \quad \text { dada por } \sigma \rightarrow \rho(\sigma)
$$

es un homomorfismo: dados $\sigma, \tau \in G$ y $v_{g} \in \mathcal{B}$ se tiene que

$$
\rho(\sigma \tau)\left(v_{g}\right)=v_{(\sigma \tau) g}=v_{\sigma(\tau g)}=\rho(\sigma)\left(v_{\tau g}\right)=\rho(\sigma)\left(\rho(\tau)\left(v_{g}\right)\right)=\rho(\sigma) \rho(\tau)\left(v_{g}\right)
$$

y asi $\rho(\sigma \tau)=\rho(\sigma) \rho(\tau)$. La representación $\rho: G \longrightarrow G L(V)$ definida en $(1)$ es llamada la representación regular de $G$ y por definición su grado es el orden de $G$.

Ejemplo 3.7 (El grupo de raíces cúbicas de la unidad). Consideremos la raíz cúbica primitiva de $1, \omega=\mathrm{e}^{\frac{2 \pi}{3} \mathrm{i}}=-\frac{1}{2}+\frac{\sqrt{3}}{2} \mathrm{i}$, la cual genera el grupo cíclico $G=\langle\omega\rangle=\left\{1, \omega, \omega^{2}\right\}$. Definamos

$$
\begin{aligned}
\rho: G & \longrightarrow G L(2, \mathbb{C}) \\
g & \longmapsto\left[\begin{array}{ll}
1 & 0 \\
0 & g
\end{array}\right]
\end{aligned}
$$

$\rho$ está bien definida, puesto que $g \neq 0$. Ahora debemos demostrar que $\rho(g h)=\rho(g) \rho(h)$ para cualesquiera $g, h \in G$. Veamos.

$$
\rho(g) \rho(h)=\left[\begin{array}{ll}
1 & 0 \\
0 & g
\end{array}\right]\left[\begin{array}{ll}
1 & 0 \\
0 & h
\end{array}\right]=\left[\begin{array}{cc}
1 & 0 \\
0 & g h
\end{array}\right]=\rho(g h) .
$$


Luego, $\rho$ es un homomorfismo de grupos y por lo tanto define una representación de $G$.

Consideremos ahora la representación de $G$ dada por

$$
\begin{aligned}
\tilde{\rho}: G & \longrightarrow G L(2, \mathbb{C}) \\
g & \longmapsto
\end{aligned}
$$

¿Cuál es la relación entre $\rho$ y $\tilde{\rho}$ ? Vamos a averiguarlo considerando para ello la matriz $P=\left[\begin{array}{ll}0 & 1 \\ 1 & 0\end{array}\right]$ cuya inversa es ella misma. Notemos que

$$
P^{-1} \tilde{\rho}(g) P=\left[\begin{array}{ll}
0 & 1 \\
1 & 0
\end{array}\right]\left[\begin{array}{ll}
g & 0 \\
0 & 1
\end{array}\right]\left[\begin{array}{ll}
0 & 1 \\
1 & 0
\end{array}\right]=\left[\begin{array}{ll}
0 & 1 \\
g & 0
\end{array}\right]\left[\begin{array}{ll}
0 & 1 \\
1 & 0
\end{array}\right]=\left[\begin{array}{ll}
1 & 0 \\
0 & g
\end{array}\right]=\rho(g)
$$

de donde por la definición 3.2, las representaciones (2) y (3) son equivalentes de grado dos (que es la dimensión del espacio $V=\mathbb{C}^{2}$ ).

Ahora se mostrarán tres maneras de operar con representaciones, obteniendo como resultado tres nuevas representaciones.

Proposición 3.8. Sean $(V, \rho)$ y $(W, \tilde{\rho})$ dos representaciones de un grupo $G$. Entonces la suma directa $V \oplus W$ y el producto tensorial $V \otimes W$ son representaciones de $G$.

Prueba. Para la suma directa consideremos $(v, w) \in V \oplus W$ y $g \in G$. Defínase

$$
\begin{aligned}
& f_{g}: V \oplus W \quad \longrightarrow \quad V \oplus W \\
& (v, w) \longmapsto(\rho(g)(v), \tilde{\rho}(g)(w))
\end{aligned}
$$

Veamos que $f_{g}$ definido como en (4) es automorfismo en $V \oplus W$.

a) $f_{g}$ es transformación lineal.

Sean $\left(v_{1}, w_{1}\right),\left(v_{2}, w_{2}\right) \in V \oplus W$ y $\lambda \in \mathbb{C}$. Luego

$$
\begin{aligned}
f_{g}\left(\left(v_{1}, w_{1}\right)+\left(v_{2}, w_{2}\right)\right) & =f_{g}\left(v_{1}+v_{2}, w_{1}+w_{2}\right) \\
& =\left(\rho(g)\left(v_{1}+v_{2}\right), \tilde{\rho}(g)\left(w_{1}+w_{2}\right)\right) \\
& =\left(\rho(g)\left(v_{1}\right)+\rho(g)\left(v_{2}\right), \tilde{\rho}(g)\left(w_{1}\right)+\tilde{\rho}(g)\left(w_{2}\right)\right) \\
& =\left(\rho(g)\left(v_{1}\right), \tilde{\rho}(g)\left(w_{1}\right)\right)+\left(\rho(g)\left(v_{2}\right), \tilde{\rho}(g)\left(w_{2}\right)\right) \\
& =f_{g}\left(v_{1}, w_{1}\right)+f_{g}\left(v_{2}, w_{2}\right) . \\
f_{g}\left(\lambda\left(v_{1}, w_{1}\right)\right) & =f_{g}\left(\lambda v_{1}, \lambda v_{2}\right) \\
& =\left(\rho(g)\left(\lambda v_{1}\right), \tilde{\rho}(g)\left(w_{1}\right)\right) \\
& =\left(\lambda \rho(g)\left(v_{1}\right), \lambda \tilde{\rho}(g)\left(w_{1}\right)\right) \\
& =\lambda\left(\rho(g)\left(v_{1}\right), \tilde{\rho}(g)\left(w_{1}\right)\right) \\
& =\lambda f_{g}\left(v_{1}, w_{1}\right) .
\end{aligned}
$$


b) $f_{g}$ es invertible.

Como $V \oplus W$ es de dimensión finita, es suficiente demostrar que $f_{g}$ es inyectiva. Para ello basta ver que ker $f_{g}=\left\{\left(0_{V}, 0_{W}\right)\right\}$.

$(v, w) \in \operatorname{ker} f_{g} \Longrightarrow f_{g}(v, w)=\left(0_{V}, 0_{W}\right) \Longrightarrow(\rho(g)(v), \tilde{\rho}(g)(w))=\left(0_{V}, 0_{W}\right)$. Luego $\rho(g)(v)=0_{V}$ y $\tilde{\rho}(g)(w)=0_{W}$; de donde $v=0_{V}$ y $w=0_{W}$ por ser $\rho(g)$ y $\tilde{\rho}(g)$ inyectivas. Así, $(v, w)=\left(0_{V}, 0_{W}\right)$ y por lo tanto ker $f_{g}=\left\{\left(0_{V}, 0_{W}\right)\right\}$.

Como $f_{g}$ es un automorfismo en $V \oplus W$, tiene sentido definir

$$
\begin{array}{ccc}
\beta: G & \longrightarrow & \mathrm{GL}(V \oplus W) \\
g & \longmapsto & f_{g}
\end{array}
$$

c) $\beta$ es un homomorfismo de grupos.

Sean $g, h \in G$ y $(v, w) \in V \oplus W$ arbitrarios. Luego

$$
\begin{aligned}
\beta(g h)(v, w) & =f_{g h}(v, w) \\
& =(\rho(g h)(v), \tilde{\rho}(g h)(w)) \\
& =(\rho(g)(\rho(h)(v)), \tilde{\rho}(g)(\tilde{\rho}(h)(w))) \\
& =f_{g}(\rho(h)(v), \tilde{\rho}(h)(w)) \\
& =f_{g}\left(f_{h}(v, w)\right) \\
& =\left(f_{g} f_{h}\right)(v, w) \\
& =(\beta(g) \beta(h))(v, w) .
\end{aligned}
$$

De $(a),(b)$ y $(c)$ concluimos que $(V \oplus W, \beta)$ es una representación de $G$ con grado igual a la suma de las dimensiones de $V$ y $W$.

Para el producto tensorial sean $\operatorname{dim} V=m, \operatorname{dim} W=n$ y $\mathcal{B}_{V}=\left\{v_{i}\right\}_{i=1}^{m}, \mathcal{B}_{W}=\left\{w_{j}\right\}_{j=1}^{n}$ bases de $V$ y $W$ respectivamente; entonces $\mathcal{B}=\left\{v_{i} \otimes w_{j}\right\}_{i=1, j=1}^{m, n}$ es una base de $V \otimes W$, de donde $\operatorname{dim} V \otimes W=m n$. Luego, el grado de la representación obtenida será $m n$.

Por otro lado, como $\rho(g): V \longrightarrow V$ y $\tilde{\rho}(g): W \longrightarrow W$ son automorfismos, podemos tensorizarlos y obtener $\rho(g) \otimes \tilde{\rho}(g): V \otimes W \longrightarrow V \otimes W$, con regla de correspondencia $(\rho(g) \otimes \tilde{\rho}(g))(v \otimes w):=\rho(g)(v) \otimes \tilde{\rho}(g)(w)$ para todo $v \otimes w \in V \otimes W$. La función anterior además satisface lo siguiente:

a) $\rho(g) \otimes \tilde{\rho}(g)$ es una transformación lineal.

$$
\text { Sean } \lambda \in \mathbb{C}_{\mathrm{y}}\left(\sum_{k=1}^{s} v_{i_{k}} \otimes w_{j_{k}}\right) \in V \otimes W \text {. Entonces }
$$


i) $\rho(g) \otimes \tilde{\rho}(g)$ separa sumas.

$$
\begin{aligned}
(\rho(g) \otimes \tilde{\rho}(g))\left(\sum_{k=1}^{s} v_{i_{k}} \otimes w_{j_{k}}\right) & =\sum_{k=1}^{s}(\rho(g) \otimes \tilde{\rho}(g))\left(v_{i_{k}} \otimes w_{j_{k}}\right) \\
& =\sum_{k=1}^{s} \rho(g)\left(v_{i_{k}}\right) \otimes \tilde{\rho}(g)\left(w_{j_{k}}\right) .
\end{aligned}
$$

ii) $\rho(g) \otimes \tilde{\rho}(g)$ saca escalares.

$$
\begin{aligned}
(\rho(g) \otimes \tilde{\rho}(g))\left(\lambda v_{i} \otimes w_{j}\right) & =\rho(g)\left(\lambda v_{i}\right) \otimes \tilde{\rho}(g)\left(w_{j}\right) \\
& =\lambda \rho(g)\left(v_{i}\right) \otimes \tilde{\rho}(g)\left(w_{j}\right) \\
& =\rho(g)\left(v_{i}\right) \otimes \lambda \tilde{\rho}(g)\left(w_{j}\right) \\
& =\lambda\left(\rho(g)\left(v_{i}\right) \otimes \tilde{\rho}(g)\left(w_{j}\right)\right) \\
& =\lambda\left((\rho(g) \otimes \tilde{\rho}(g))\left(v_{i} \otimes w_{j}\right)\right)
\end{aligned}
$$

b) $\rho(g) \otimes \tilde{\rho}(g)$ es invertible.

Como $\operatorname{dim} V \oplus W=(\operatorname{dim} V)(\operatorname{dim} W)=m n<\infty$, es suficiente demostrar la suprayectividad de $\rho(g) \otimes \tilde{\rho}(g)$ ya que la inyectividad será automática.

Sea $x \in V \otimes W$; entonces $x=\sum_{k=1}^{s} \lambda_{k} v_{i_{k}} \otimes w_{j_{k}}$, donde $\lambda_{k} \in \mathbb{C}, v_{i_{k}} \in \mathcal{B}_{V}$ y $w_{j_{k}} \in \mathcal{B}_{W}$. Como $v_{i_{k}} \in V$ y $\rho(g): V \longrightarrow V$ es invertible (y en particular es sobre), existe $\tilde{v}_{i_{k}} \in V$ tal que $\rho(g)\left(\tilde{v}_{i_{k}}\right)=v_{i_{k}}$. Análogamente, como $\tilde{\rho}(g): W \longrightarrow W$ es sobre, existe $\tilde{w}_{j_{k}} \in W$ tal que $\tilde{\rho}(g)\left(\tilde{w}_{j_{k}}\right)=w_{j_{k}}$. Se define $y:=\sum_{k=1}^{s} \lambda_{k} \tilde{v}_{i_{k}} \otimes \tilde{w}_{j_{k}}$. Luego

$$
\begin{aligned}
(\rho(g) \otimes \tilde{\rho}(g))(y) & =(\rho(g) \otimes \tilde{\rho}(g))\left(\sum_{k=1}^{s} \lambda_{k} \tilde{v}_{i_{k}} \otimes \tilde{w}_{j_{k}}\right) \\
& =\sum_{k=1}^{s}(\rho(g) \otimes \tilde{\rho}(g))\left(\lambda_{k} \tilde{v}_{i_{k}} \otimes \tilde{w}_{j_{k}}\right) \\
& =\sum_{k=1}^{s} \rho(g)\left(\lambda_{k} \tilde{v}_{i_{k}}\right) \otimes \tilde{\rho}(g)\left(\tilde{w}_{j_{k}}\right) \\
& =\sum_{k=1}^{s} \lambda_{k} \rho(g)\left(\tilde{v}_{i_{k}}\right) \otimes \tilde{\rho}(g)\left(\tilde{w}_{j_{k}}\right) \\
& =\sum_{k=1}^{s} \lambda_{k} v_{i_{k}} \otimes w_{j_{k}} \\
& =x .
\end{aligned}
$$

En consecuencia, $\rho(g) \otimes \tilde{\rho}(g)$ es sobre y, por lo tanto, invertible. 
De $(a)$ y $(b)$ concluimos que $\rho(g) \otimes \tilde{\rho}(g)$ es un automorfismo, por lo que podemos definir

$$
\begin{aligned}
\gamma: G & \longrightarrow \mathrm{GL}(V \otimes W) \\
g & \longmapsto \rho(g) \otimes \tilde{\rho}(g)
\end{aligned}
$$

c) $\gamma$ es homomorfismo de grupos.

$$
\begin{aligned}
& \text { Sean } g, h \in G \text { y } \sum_{k=1}^{s} \lambda_{k} v_{i_{k}} \otimes w_{j_{k}} \in V \otimes W \text { arbitrarios. Entonces } \\
& \gamma(g h)\left(\sum_{k=1}^{s} \lambda_{k} v_{i_{k}} \otimes w_{j_{k}}\right)=(\rho(g h) \otimes \tilde{\rho}(g h))\left(\sum_{k=1}^{s} \lambda_{k} v_{i_{k}} \otimes w_{j_{k}}\right) \\
& =\sum_{k=1}^{s}(\rho(g h) \otimes \tilde{\rho}(g h))\left(\lambda_{k} v_{i_{k}} \otimes w_{j_{k}}\right) \\
& =\sum_{k=1}^{s} \rho(g h)\left(\lambda_{k} v_{i_{k}}\right) \otimes \tilde{\rho}(g h)\left(w_{j_{k}}\right) \\
& =\sum_{k=1}^{s} \rho(g)\left(\rho(h)\left(\lambda_{k} v_{i_{k}}\right)\right) \otimes \tilde{\rho}(g)\left(\tilde{\rho}(h)\left(w_{j_{k}}\right)\right) \\
& =\sum_{k=1}^{s}(\rho(g) \otimes \tilde{\rho}(g))\left(\rho(h)\left(\lambda_{k} v_{i_{k}}\right) \otimes \tilde{\rho}(h)\left(w_{j_{k}}\right)\right) \\
& =(\rho(g) \otimes \tilde{\rho}(g))\left(\sum_{k=1}^{s} \rho(h)\left(\lambda_{k} v_{i_{k}}\right) \otimes \tilde{\rho}(h)\left(w_{j_{k}}\right)\right) \\
& =\gamma(g)\left(\sum_{k=1}^{s}(\rho(h) \otimes \tilde{\rho}(h))\left(\lambda_{k} v_{i_{k}} \otimes w_{j_{k}}\right)\right) \\
& =\gamma(g)\left((\rho(h) \otimes \tilde{\rho}(h))\left(\sum_{k=1}^{s} \lambda_{k} v_{i_{k}} \otimes w_{j_{k}}\right)\right) \\
& =\gamma(g)\left(\gamma(h)\left(\sum_{k=1}^{s} \lambda_{k} v_{i_{k}} \otimes w_{j_{k}}\right)\right) \\
& =(\gamma(g) \circ \gamma(h))\left(\sum_{k=1}^{s} \lambda_{k} v_{i_{k}} \otimes w_{j_{k}}\right)
\end{aligned}
$$

De $(a),(b)$ y $(c)$, concluimos que $(V \otimes W, \gamma)$ es una representación de $G$.

Proposición 3.9. Sea $(V, \rho)$ una representación de un grupo $G$. Entonces, la $\boldsymbol{n}$-ésima potencia tensorial de $V$, denotada como $V^{\otimes n}:=\underbrace{V \otimes V \otimes \cdots \otimes V}_{n \text {-veces }}$ admite una estructura de representación. 
Prueba. Si $\operatorname{dim} V=d$, entonces $\operatorname{dim} V^{\otimes n}=d^{n}$. Considérese la función

$$
\begin{aligned}
\delta: G & \longrightarrow \mathrm{GL}\left(V^{\otimes n}\right) \\
g & \longmapsto \rho(g)^{\otimes n}
\end{aligned}
$$

donde $\rho(g)^{\otimes n}:=\underbrace{\rho(g) \otimes \cdots \otimes \rho(g)}_{n \text {-veces }}$. Esta definición hace a $\delta$ un homomorfismo de grupos y a $\rho^{\otimes n}$ un automorfismo de $V^{\otimes n}$. Por la definición 3.1, $\left(V^{\otimes}, \delta\right)$ es una representación de $G$.

Una pregunta natural que podría uno hacerse es la siguiente: ¿un subespacio vectorial $W$ puede heredar la estructura de representación del espacio ambiente $V$ ? La respuesta es sí y la siguiente definición establece la condición que tal subespacio ha de cumplir.

Definición 3.10. Una sub-representación de una representación $(V, \rho)$ es un subespacio vectorial $W \subset V$ que es invariante bajo $G$ (o G-invariante). Es decir

$$
\rho(g) W \subset W, \quad \forall g \in G
$$

Ahora se presentan dos ejemplos sencillos de sub-representaciones.

Ejemplo 3.11. Los subespacios 0 y $V$ de un espacio vectorial $V$ son $G$-invariantes, y se denominan sub-representaciones triviales del grupo $G$.

Ejemplo 3.12. Sean $(V, \rho)$ y $(W, \tilde{\rho})$ dos representaciones de un grupo $G$, y sea $\varphi: V \longrightarrow W$ un morfismo de representaciones (véase la definición 3.1). Entonces ker $\varphi$ e $\operatorname{Im} \varphi$ son subrepresentaciones de $V$ y $W$ respectivamente.

Prueba. Del álgebra lineal sabemos que $\operatorname{ker} \varphi<V \operatorname{e} \operatorname{Im} \varphi<W$.

(a) $\rho(g)(\operatorname{ker} \varphi) \subset \operatorname{ker} \varphi, \forall g \in G$.

Sean $v \in \operatorname{ker} \varphi$ y $g \in G$ arbitrarios. Luego

$\varphi(\rho(g)(v))=(\varphi \rho(g))(v)=(\tilde{\rho}(g) \varphi)(v)=\tilde{\rho}(g)(\varphi(v))=\tilde{\rho}(g)\left(0_{\tilde{V}}\right)=0_{\tilde{V}}$, de modo que $\rho(g)(v) \in \operatorname{ker} \varphi$. Por lo tanto, $\rho(g)(\operatorname{ker} \varphi) \subset \operatorname{ker} \varphi$.

(b) $\tilde{\rho}(g)(\operatorname{Im} \varphi) \subset \operatorname{Im} \varphi$.

Sean $w \in \operatorname{Im} \varphi$ y $g \in G$ arbitrarios. Como $w \in \operatorname{Im} \varphi$, existe $v \in V$ tal que $\varphi(v)=w$. Así que $\tilde{\rho}(g)(w)=\tilde{\rho}(g)(\varphi(v))=(\tilde{\rho}(g) \varphi)(v)=(\varphi \rho(g))(v)=\varphi(\rho(g)(v))$. Por consiguiente $\tilde{\rho}(g)(w)=\varphi(\rho(g)(w))$, de donde $\tilde{\rho}(g)(w) \in \operatorname{Im} \varphi$. Por lo tanto, $\tilde{\rho}(g)(\operatorname{Im} \varphi) \subset \operatorname{Im} \varphi$.

Definición 3.13. Una representación $V \neq 0$ se dirá irreducible si no existe ningún subespacio propio invariante $W$ de $V$ distinto del trivial (dicho de otra manera: sus únicas subrepresentaciones son 0 y $V$ mismo), y la llamaremos indescomponible si no se puede expresar, a su vez, como una suma directa de sub-representaciones no nulas (i.e., si $V=V_{1} \oplus V_{2}$, entonces $V_{1}=0$ ó $\left.V_{2}=0\right)$. 
Ejemplo 3.14. Sean $V=\mathbb{C}^{2}, G=\left\{1, \omega, \omega^{2}\right\}$ y $\rho$ definidos como en el ejemplo $3.7 y$ consideremos el subespacio $W$ de $V$ definido como

$$
W=\left\{\left[\begin{array}{l}
w \\
0
\end{array}\right]: w \in \mathbb{C}\right\}
$$

Luego

$$
\rho(g) W=\left[\begin{array}{ll}
1 & 0 \\
0 & g
\end{array}\right]\left[\begin{array}{l}
w \\
0
\end{array}\right]=\left[\begin{array}{l}
w \\
0
\end{array}\right] \in W \quad \forall g \in G,\left[\begin{array}{l}
w \\
0
\end{array}\right] \in W
$$

de donde $\rho(g) W \subset W$ y por lo tanto $W$ es $G$-invariante. De la definición 3.10 se sigue que $W$ es una sub-representación no trivial de $V$, lo cual prueba que la representación $\rho$ definida en la ecuación (2) no es irreducible (o sea, es reducible). Ya para terminar, probaremos que $\rho$ tampoco es indescomponible (o sea, es descomponible).

El subespacio complementario $W^{\prime}$ de $W$ es

$$
W^{\prime}=\left\langle\left[\begin{array}{l}
0 \\
1
\end{array}\right]\right\rangle=\left\{\left[\begin{array}{l}
0 \\
z
\end{array}\right]: z \in \mathbb{C}\right\} \text {. }
$$

Luego

$$
\rho(g) W^{\prime}=\left[\begin{array}{ll}
1 & 0 \\
0 & g
\end{array}\right]\left[\begin{array}{l}
0 \\
z
\end{array}\right]=\left[\begin{array}{c}
0 \\
g z
\end{array}\right] \in W^{\prime} \quad \forall g \in G,\left[\begin{array}{l}
0 \\
z
\end{array}\right] \in W^{\prime}
$$

de donde $\rho(g) W^{\prime} \subset W^{\prime}$ y por lo tanto $W^{\prime}$ es $G$-invariante; i.e., $W^{\prime}$ es también una subrepresentación de $V$ no trivial. De (5) y (6) obtenemos finalmente la descomposición deseada

$$
V=W \oplus W^{\prime} .
$$

Por otro lado, de la definición 3.13 se deduce inmediatamente que irreducible implica indescomponible, lo cual es cierto aun si el grupo $G$ es infinito. El recíproco es válido en grupos finitos, pero no necesariamente en grupos infinitos. Veamos un contraejemplo.

Ejemplo 3.15 (Una representación indescomponible que no es irreducible). Sea $\mathbb{Z}$ el grupo aditivo de los enteros y definamos

$$
\begin{aligned}
\rho: \mathbb{Z} & \longrightarrow G L(2, \mathbb{C}) \\
n & \longmapsto\left[\begin{array}{ll}
1 & n \\
0 & 1
\end{array}\right]
\end{aligned}
$$

Como el determinante de la matriz dada en (7) es distinto de cero, se sigue que dicha matriz es invertible y por tanto $\rho$ está bien definido. Notemos que

$$
\rho(m) \rho(n)=\left[\begin{array}{cc}
1 & m \\
0 & 1
\end{array}\right]\left[\begin{array}{cc}
1 & n \\
0 & 1
\end{array}\right]=\left[\begin{array}{cc}
1 & m+n \\
0 & 1
\end{array}\right]=\rho(m+n), \quad \forall m, n \in \mathbb{Z} .
$$

Luego, $\rho$ es un homomorfismo de grupos y, en consecuencia, una representación de $\mathbb{Z}$. 
Considere ahora el subespacio propio $W$ de $\mathbb{C}^{2}$ definido como

$$
W=\left\{\left[\begin{array}{l}
w \\
0
\end{array}\right]: w \in \mathbb{C}\right\} .
$$

No es dificil probar que $\rho(n)(W) \subset W$ para todo entero $n$; es decir, $W$ es $\mathbb{Z}$-invariante $y$ define así una sub-representación no trivial de $\mathbb{Z}$. En consecuencia, $\rho$ no es irreducible.

Ahora bien, si $W^{\prime} \subset \mathbb{C}^{2}$ es un subespacio complementario de $W$ definido en (8), entonces es de la forma

$$
W^{\prime}=\left\langle\left[\begin{array}{l}
\lambda \\
1
\end{array}\right]\right\rangle=\left\{\left[\begin{array}{c}
\lambda z \\
z
\end{array}\right]: z \in \mathbb{C}\right\}
$$

fijando un $\lambda \in \mathbb{C}$ elegido arbitrariamente. Empero, $W^{\prime}$ no es $\mathbb{Z}$-invariante y por lo tanto no es una sub-representación de $\mathbb{Z}$. En consecuencia, $\rho$ es indescomponible.

Un resultado ligado a la definición 3.13 es el siguiente: cualquier representación es una suma directa de representaciones irreducibles. Esta propiedad es llamada reducibilidad completa o semisimplicidad [4, pág. 6].

Lema 3.16 (Schur). Sean $(V, \rho),(W, \tilde{\rho})$ dos representaciones irreducibles de un grupo $G$ y $\varphi: V \longrightarrow W$ un homomorfismo de G-módulos. Entonces

1) O bien $\varphi$ es un isomorfismo, o bien $\varphi=0$.

2) Si $V=W$, entonces $\varphi=\lambda I$ para algún $\lambda \in \mathbb{C}$, I la identidad.

Prueba. Detallaremos las ideas de Fulton y Harris [4, pág. 7], teniendo en cuenta que ker $\varphi$ e $\operatorname{Im} \varphi$ son sub-representaciones de $V$ y $W$ respectivamente (véase 3.12).

1) $\operatorname{Si} \varphi=0$ no hay nada que demostrar. Supongamos entonces que $\varphi \neq 0$. De aquí se sigue que $\operatorname{ker} \varphi$ no puede ser igual a $V$, pero al ser éste irreducible se tiene que $\operatorname{ker} \varphi=0_{V}$, por lo que $\varphi$ es inyectiva. Análogamente, como $\varphi \neq 0$ entonces $\operatorname{Im} \varphi \neq 0_{W}$. Pero $W$ es irreducible, de donde $\operatorname{Im} \varphi=W$ y $\varphi$ es sobre. En consencuencia $\varphi$ es isomorfismo.

2) Si $V=W$ entonces $\varphi$ es un endomorfismo en $V$. Como $\mathbb{C}$ es algebraicamente cerrado, $\varphi$ tiene al menos un autovalor $\lambda$. Es decir, para algún $\lambda \in \mathbb{C}, \operatorname{ker}(\varphi-\lambda I) \neq 0$. Por el inciso (1) anterior tenemos que $\varphi-\lambda I=0_{V}$, y en consecuencia $\varphi=\lambda I$.

El resultado que sigue establece la existencia y unicidad de una descomposición en irreducibles de una representación. Veamos.

Proposición 3.17. Para cualquier representación $(V, \rho)$ de un grupo finito $G$, existe una descomposición

$$
V=V_{1}^{\oplus a_{1}} \oplus \cdots \oplus V_{k}^{\oplus a_{k}}
$$

donde las $V_{i}$ son representaciones irreducibles distintas. La descomposición de $V$ como una suma directa de los $k$ sumandos es única, como lo son las $V_{i}$ que aparecen y sus multiplicidades $a_{i}$. 
Prueba. (Cfr. [4, pág. 6]) Sea $W$ otra representación de $G$ con descomposición en irreducibles dada por $W=\oplus W_{j}^{\oplus b_{j}}$ y $\varphi: V \longrightarrow W$ un morfismo de representaciones. Por el lema de Schur, $\varphi$ manda el factor $V_{i}^{\oplus a_{i}}$ al factor $W_{j}^{\oplus b_{j}}$, de donde $W_{j} \cong V_{i}$; aplicando entonces la función identidad de $V$ en $V$, la unicidad se sigue.

¿La cantidad de representaciones irreducibles de un grupo $G$ es finita? La respuesta es sí: el número de representaciones irreducibles de $G$, salvo isomorfismo, es igual al número de clases de conjugación de $G$ [8, pág. 196, (2)]. Ahora bien, ¿es posible determinar con exactitud este número? En teoría sí, pero esta labor se vuelve muy pesada cuanto más grande sea el orden de $G$. No obstante, una herramienta útil en el cálculo de tal número es la de partición, misma que se define en la siguiente subsección.

\subsection{El Simetrizador de Young}

Definición 3.18. Una partición $\lambda$ de un entero positivo $n$, denotada por $\lambda \vdash n$, es una $r$-ada $\lambda=\left(\lambda_{1}, \lambda_{2}, \ldots, \lambda_{r}\right)$ que satisface las siguientes condiciones:

(a) $\lambda_{i} \in \mathbb{N} \quad \forall i=1,2, \ldots, r \quad\left(\right.$ cada $\lambda_{i}$ es un entero positivo $\left.{ }^{2}\right)$.

(b) $\lambda_{1} \geq \lambda_{2} \geq \cdots \geq \lambda_{r} \quad$ (la sucesión de $\lambda_{i}$ 's es no creciente).

(c) $\sum_{i=1}^{r} \lambda_{i}=\lambda_{1}+\lambda_{2}+\cdots+\lambda_{r}=n \quad$ (la suma de las $\lambda_{i}$ 's nos da el natural $n$ ).

Las particiones $\alpha=(n)$ y $\beta=\left(1^{n}\right)=(\underbrace{1, \ldots, 1}_{n-v e c e s})$ se denominan particiones triviales de $n$.

El Teorema de Cayley (1854) afirma que: si $G$ es un grupo finito de orden $n$, entonces es isomorfo a un subgrupo del grupo simétrico $S_{n}$. Como el número de clases de conjugación de $S_{n}$ es igual al número de particiones de $n$ [8, pág. 45], la tarea se "reduce" a calcular (ó estimar) con la mayor exactitud posible la función partición $p(n)$.

Valores pequeños de $n$ no representan mayor problema: $p(1)=1, p(3)=3, p(5)=7$. Luego la sucesión comienza a crecer muy rápido: $p(10)=42, p(30)=5604, p(50)=204,226$, $p(100)=190,569,292$, etc. Pero, aun cuando no se tiene una fórmula práctica para calcular el número exacto de particiones de un natural $n$, existe una expresión asintótica de la función partición $p(n)$ obtenida por los matemáticos G.H. Hardy y S. Ramanujan en 1918 [5]:

$$
p(n) \sim \frac{\exp \left(\pi \sqrt{\frac{2 n}{3}}\right)}{4 n \sqrt{3}} \text { cuando } n \rightarrow \infty .
$$

\footnotetext{
${ }^{2}$ En ocasiones resulta conveniente admitir entradas nulas, como cuando se define un orden parcial en las particiones, v.g. la inclusión: dadas $\mu=\left(\mu_{1}, \ldots, \mu_{r}\right)$ y $\nu=\left(\nu_{1}, \ldots, \nu_{s}\right)$ particiones de ciertos enteros, diremos que $\mu \subset \nu$ si $\mu_{i} \leq \nu_{i}$ para toda $i=1, \ldots, r$. De aquí que, si $r \leq s-1$ entonces se considera $\mu_{i}=0$ para $i=r+1, \ldots, s$. Así, el número de entradas de ambas particiones queda igualado.
} 
Definición 3.19. Considere una partición $\lambda \vdash n$, entonces:

(1) Un diagrama de Young, denotado por $[\lambda]$, es el conjunto:

$$
[\lambda]=\left\{(i, j): i, j \in \mathbb{N}, 1 \leq i, 1 \leq j \leq \lambda_{i}\right\}
$$

donde el par $(i, j) \in[\lambda]$ se denomina nodo de $\lambda$.

(2) Un tablero $T_{\lambda}$ es uno de los $n$ ! arreglos obtenidos por la sustitución de cada nodo en $[\lambda]$ por uno de los enteros del conjunto $\{1,2, \ldots, n\}$, sin repetición.

Veamos dos ejemplos ilustrativos para la definición precedente.

Ejemplo 3.20. Sea $\lambda=(2,2)$ una partición de $n=4$. Su diagrama de Young correspondiente es $[\lambda]=\{(1,1),(1,2),(2,1),(2,2)\}$.
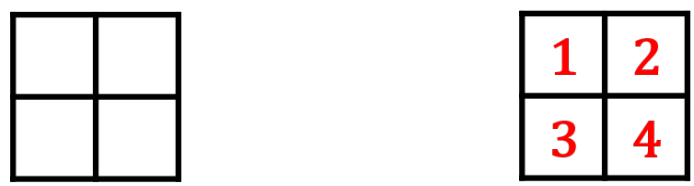

Figura 1: Diagrama de Young (izq.) y tablero (der.) asociados a $\lambda=(2,2)$.

Ejemplo 3.21. Sea $\lambda=(4,3,1) \vdash 8$. El diagrama de Young que corresponde a esta partición es $[\lambda]=\{(1,1),(1,2),(1,3),(1,4),(2,1),(2,2),(2,3),(3,1)\}$.


Figura 2: Diagrama de Young (izq.) y tablero (der.) asociados a $\lambda=(4,3,1)$.

Antes de continuar, será necesario introducir las tres definiciones que siguen.

Definición 3.22. El álgebra de grupo $\mathbb{C} G$ se define como el espacio vectorial que tiene como base el grupo $G$ :

$$
\mathbb{C} G=\left\{\sum_{g \in G} z_{g} \cdot g \mid g \in G, z_{g} \in \mathbb{C}\right\}
$$

con un producto que resulta de extender por linealidad el producto de G:

$$
\left(\sum_{g \in G} z_{g} \cdot g\right)\left(\sum_{h \in G} w_{h} \cdot h\right)=\sum_{t \in G}\left(\sum_{g h=t} z_{g} w_{h}\right) t
$$

y que lo convierte en una $\mathbb{C}$-álgebra. Este producto se puede definir en básicos $\left\{e_{g}: g \in G\right\}$ de manera que verifiquen la igualdad $e_{g} e_{h}=e_{g h}$. 
Definición 3.23. Sean $V$ un $\mathbb{C}$-espacio vectorial de dimensión finita d y $W_{S}$ el subespacio vectorial de la potencia tensorial $V^{\otimes n}$ (véase 3.9) dado por

$$
W_{S}=\left\langle\left\{v_{1} \otimes \cdots \otimes v_{n}-v_{\sigma(1)} \otimes \cdot v_{\sigma(n)} \mid v_{i} \in V, \quad \forall i=1, \ldots, n \quad \forall \sigma \in S_{n}\right\}\right\rangle .
$$

Se define entonces la $\boldsymbol{n}$-ésima potencia tensorial simétrica, denotada por $S^{n} m^{n} V$, como el espacio vectorial cociente

$$
\operatorname{Sym}^{n} V:=\frac{V^{\otimes n}}{W_{S}}
$$

el cual es un subespacio vectorial de $V^{\otimes n}$ de dimensión $\left(\begin{array}{c}d+n-1 \\ n\end{array}\right)$.

Nótese que si $n=1$, entonces $\operatorname{dim} \operatorname{Sym}^{1} V=\left(\begin{array}{c}d+1-1 \\ 1\end{array}\right)=\left(\begin{array}{l}d \\ 1\end{array}\right)=d=\operatorname{dim} V$ y por lo tanto $\operatorname{Sym}^{1} V \cong V$. Para $n=0$, por convención se considera $\operatorname{Sym}^{0} V:=\mathbb{C}$.

Definición 3.24. Sean $V$ un $\mathbb{C}$-espacio vectorial de dimensión finita d y $W_{A}$ el subespacio vectorial de la potencia tensorial $V^{\otimes n}$ (véase 3.9) dado por

$$
W_{A}=\left\langle\left\{v_{1} \otimes \cdots \otimes v_{n} \mid v_{i}=v_{j} \quad \text { para algún } i \neq j\right\}\right\rangle .
$$

Se define entonces la n-ésima potencia tensorial exterior (o potencia tensorial alternante), denotada por $\bigwedge^{n} V$, como el espacio vectorial cociente

$$
\bigwedge^{n} V:=\frac{V^{\otimes n}}{W_{A}}
$$

el cual es un subespacio vectorial de $V^{\otimes n}$ de dimensión $\left(\begin{array}{l}d \\ n\end{array}\right)$.

Nótese que si $n=1$, entonces $\operatorname{dim} \bigwedge^{1} V=\left(\begin{array}{l}d \\ 1\end{array}\right)=d=\operatorname{dim} V$ y por lo tanto $\bigwedge^{1} V \cong V$. Para $n=0$, por convención se considera $\bigwedge^{0} V:=\mathbb{C}$ y en particular $\bigwedge^{n} V=0$ para $n>d$.

Dados una partición $\lambda=\left(\lambda_{1}, \ldots, \lambda_{k}\right) \vdash n,[\lambda]$ su diagrama de Young y $T_{\lambda}$ un tablero asociado a éste, se introducen los siguientes dos subgrupos del grupo simétrico $S_{n}$ :

$$
P_{\lambda}:=\left\{\sigma \in S_{n} \mid \sigma \text { preserva cada fila de } T_{\lambda}\right\}
$$

y

$$
Q_{\lambda}:=\left\{\tau \in S_{n} \mid \tau \text { preserva cada columna de } T_{\lambda}\right\},
$$

los cuales están bien definidos porque al menos la permutación identidad pertenece a ambos.

Por ejemplo, para la partición $\lambda=(2,2) \vdash 4$ y el tablero del Ejemplo 3.20, el subgrupo $P_{(2,2)}$ que se obtiene es

$$
P_{(2,2)}=\left\{\mathrm{id},\left(\begin{array}{ll}
1 & 2
\end{array}\right),\left(\begin{array}{ll}
3 & 4
\end{array}\right),\left(\begin{array}{ll}
1 & 2
\end{array}\right)(34)\right\}
$$

ya que $\sigma(\{1,2\})=\{1,2\}$ y $\sigma(\{3,4\})=\{3,4\}$ para toda $\sigma \in P_{(2,2)}$ (este es el significado en la definición (14) de la frase " $\sigma$ preserva cada fila de $T_{\lambda}$ "). En tanto, el subgrupo $Q_{(2,2)}$ es

$$
Q_{(2,2)}=\left\{\mathrm{id},\left(\begin{array}{ll}
1 & 3
\end{array}\right),\left(\begin{array}{ll}
2 & 4
\end{array}\right),\left(\begin{array}{ll}
1 & 3
\end{array}\right)\left(\begin{array}{ll}
2 & 4
\end{array}\right)\right\}
$$


dado que $\tau(\{1,3\})=\{1,3\}$ y $\tau(\{2,4\})=\{2,4\}$ para toda $\tau \in Q_{(2,2)}$ (a esto se refiere en la definición (15) la frase " $\tau$ preserva cada cada columna de $T_{\lambda}$ ").

Ahora, en el álgebra de grupo $\mathbb{C} S_{n}$ (véase 3.22) se introducen dos elementos correspondientes a los subgrupos $P_{\lambda}$ y $Q_{\lambda}$ definidos en (14) y (15) respectivamente:

$$
a_{\lambda}=\sum_{\sigma \in P_{\lambda}} e_{\sigma} \quad \text { y } \quad b_{\lambda}=\sum_{\tau \in Q_{\lambda}} \operatorname{sgn}(\tau) e_{\tau} .
$$

La imagen del elemento $a_{\lambda} \in \mathbb{C} S_{n}$ sobre $V^{\otimes n}$ es el subespacio

$$
\operatorname{Im}\left(a_{\lambda} \mid V^{\otimes n}\right):=\operatorname{Sym}^{\lambda_{1}} V \otimes \operatorname{Sym}^{\lambda_{2}} V \otimes \cdots \otimes \operatorname{Sym}^{\lambda_{k}} V \hookrightarrow V^{\otimes n}
$$

donde la inclusión de la derecha se obtiene reagrupando los factores de $V^{\otimes n}$ de acuerdo con las filas del tablero $T_{\lambda}$.

Análogamente, la imagen de $b_{\lambda}$ sobre $V^{\otimes n}$ está dada por el subespacio

$$
\operatorname{Im}\left(b_{\lambda} \mid V^{\otimes n}\right):=\bigwedge^{\mu_{1}} V \otimes \bigwedge^{\mu_{2}} V \otimes \cdots \otimes \bigwedge^{\mu_{s}} V \hookrightarrow V^{\otimes n}
$$

donde $\mu=\left(\mu_{1}, \ldots, \mu_{s}\right)$ es la partición conjugada ${ }^{3}$ de $\lambda$.

Finalmente, haciendo el producto de $a_{\lambda}$ y $b_{\lambda}$ dados en (18) se obtiene

$$
c_{\lambda}:=a_{\lambda} \cdot b_{\lambda} \in \mathbb{C} S_{n}
$$

llamado simetrizador de Young, cuya imagen sobre $V^{\otimes n}$ es el subespacio

$$
\operatorname{Im}\left(c_{\lambda} \mid V^{\otimes n}\right):=\left(\mathbb{C} S_{n}\right) \cdot c_{\lambda}
$$

Ejemplo 3.25. (1) Sea $\lambda=\left(\lambda_{1}\right)=(n)$; entonces $P_{(n)}=S_{n}$ (ya que todo $S_{n}$ fija la única fila de cualquier tablero $\left.T_{(n)}\right)$ y $Q_{(n)}=\{i d\}$ (por una razón análoga). Luego

$$
a_{(n)} \stackrel{(18)}{=} \sum_{\sigma \in P_{(n)}} e_{\sigma}=\sum_{\sigma \in S_{n}} e_{\sigma} \quad y \quad b_{(n)} \stackrel{(18)}{=} \sum_{\tau \in S_{n}} \operatorname{sgn}(\tau) e_{\tau}=e_{i d}
$$

de donde $c_{(n)}=a_{(n)} \cdot b_{(n)}=\left(\sum_{\sigma \in S_{n}} e_{\sigma}\right) \cdot e_{i d}=\sum_{\sigma \in S_{n}} e_{\sigma}=a_{(n)}$.

Sea $v \in \mathbb{C} S_{n}$ arbitrario, entonces es de la forma $v \stackrel{(10)}{=} \sum_{\theta \in S_{n}} z_{\theta} e_{\theta} \in \mathbb{C} S_{n}$. Luego

$$
v \cdot c_{(n)}=v \cdot a_{(n)}=\left(\sum_{\theta \in S_{n}} z_{\theta} e_{\theta}\right)\left(\sum_{\sigma \in S_{n}} e_{\sigma}\right) \stackrel{(11)}{=} \sum_{\rho \in S_{n}}\left(\sum_{\theta \sigma=\rho} z_{\theta}\right) e_{\rho}=\underbrace{\left(\sum_{\theta \in S_{n}} z_{\theta}\right)}_{\in \mathbb{C}} \sum_{\rho \in S_{n}} e_{\rho}
$$

donde la última igual se tiene porque $\sum_{\theta \sigma=\rho} z_{\theta}=\sum_{\theta \in S_{n}} z_{\theta}$, pues dado $\theta \in S_{n}$ y fijando $\rho$ se sigue que $\sigma=\theta^{-1} \rho \in S_{n}$.

Por lo tanto, $\operatorname{Im}\left(c_{\lambda} \mid V^{\otimes n}\right)=\operatorname{Im}\left(a_{\lambda} \mid V^{\otimes n}\right) \stackrel{(19)}{=} \operatorname{Sym}^{\lambda_{1}} V=\operatorname{Sym}^{n} V$.

\footnotetext{
${ }^{3}$ Una partición $\lambda^{\prime}$ se dirá conjugada de $\lambda$ cuando se intercambian las filas por las columnas en el diagrama de Young asociado a $\lambda$.
} 
(2) Si $\lambda=\left(1^{n}\right)$, su conjugada es $\mu=\left(\mu_{1}\right)=(n)$. Entonces $P_{\left(1^{n}\right)}=\{i d\}$ y $Q_{\left(1^{n}\right)}=S_{n}(y a$ que todo $S_{n}$ preserva la única columna de cualquier tablero $\left.T_{\left(1^{n}\right)}\right)$. Luego

$$
a_{\left(1^{n}\right)}=\sum_{\sigma \in P_{\left(1^{n}\right)}} e_{\sigma}=e_{i d} \quad y \quad b_{\left(1^{n}\right)}=\sum_{\tau \in Q_{\left(1^{n}\right)}} \operatorname{sgn}(\tau) e_{\tau}=\sum_{\tau \in S_{n}} \operatorname{sgn}(\tau) e_{\tau}
$$

de donde $c_{\left(1^{n}\right)}=a_{\left(1^{n}\right)} \cdot b_{\left(1^{n}\right)}=e_{i d} \cdot\left(\sum_{\tau \in S_{n}} \operatorname{sgn}(\tau) e_{\tau}\right)=\sum_{\tau \in S_{n}} \operatorname{sgn}(\tau) e_{\tau}=b_{\left(1^{n}\right)}$.

Sea $v \in \mathbb{C} S_{n}$ arbitrario, entonces es de la forma $v \stackrel{(10)}{=} \sum_{\theta \in S_{n}} z_{\theta} e_{\theta} \in \mathbb{C} S_{n}$. Luego

$$
\begin{aligned}
v \cdot c_{\left(1^{n}\right)} & =v \cdot b_{\left(1^{n}\right)} \\
& =\left(\sum_{\theta \in S_{n}} z_{\theta} e_{\theta}\right)\left(\sum_{\tau \in S_{n}} \operatorname{sgn}(\tau) e_{\tau}\right) \\
& \stackrel{(11)}{=} \sum_{\rho \in S_{n}}\left(\sum_{\theta \tau=\rho} \operatorname{sgn}(\tau) z_{\tau}\right) e_{\rho} \\
& =\sum_{\rho \in S_{n}}\left(\sum_{\theta \in S_{n}} \operatorname{sgn}\left(\theta^{-1} \rho\right) z_{\tau}\right) e_{\rho} \\
& =\underbrace{\left(\sum_{\theta \in S_{n}} \operatorname{sgn}\left(\theta^{-1}\right) z_{\tau}\right)}_{\in \mathbb{C}}\left(\sum_{\rho \in S_{n}} \operatorname{sgn}(\rho) e_{\rho}\right) .
\end{aligned}
$$

En consecuencia, $\operatorname{Im}\left(c_{\lambda} \mid V^{\otimes n}\right)=\operatorname{Im}\left(b_{\lambda} \mid V^{\otimes n}\right) \stackrel{(20)}{=} \bigwedge^{\mu_{1}} V=\bigwedge^{n} V$.

Teorema 3.26. [4, pág. 46] Algún múltiplo escalar de $c_{\lambda}$ es idempotente (es decir, $c_{\lambda}^{2}=$ $\left.n_{\lambda} c_{\lambda}\right)$, y la imagen de $c_{\lambda}$ es una representación irreducible $V_{\lambda}$ de $S_{n}$. Cada representación irreducible de $S_{n}$ se puede obtener de esta forma para una única partición.

La importancia del teorema 3.26 estriba en que da una correspondencia implícita (pero directa) entre las clases de conjugación del grupo simétrico $S_{n}$ y las representaciones irreducibles del mismo, algo que no se ha logrado para grupos en general.

Ejemplo 3.27. Consideremos la partición $\lambda=(2,2) \vdash 4$ y el tablero dados en 3.20, y los subgrupos $P_{(2,2)}$ y $Q_{(2,2)}$ de $S_{4}$ descritos en (16) y (17) respectivamente. Entonces

$$
\begin{aligned}
& a_{(2,2)} \stackrel{\stackrel{(18)}{=}}{=} \sum_{\sigma \in P_{(2,2)}} e_{\sigma}=e_{i d}+e_{(12)}+e_{(34)}+e_{(12)(34)} . \\
& b_{(2,2)} \stackrel{(18)}{=} \sum_{\tau \in Q_{(2,2)}} \operatorname{sgn}(\tau) e_{\tau}=e_{i d}-e_{(13)}-e_{(24)}+e_{(13)(24)}
\end{aligned}
$$


de donde

$$
\begin{array}{cll}
c_{(2,2)} & \stackrel{(21)}{=} & a_{(2,2)} \cdot b_{(2,2)} \\
& \stackrel{(23,24)}{=} & \left(e_{i d}+e_{(12)}+e_{(34)}+e_{(12)(34)}\right) \cdot\left(e_{i d}-e_{(13)}-e_{(24)}+e_{(13)(24)}\right) \\
= & e_{i d}+e_{(12)}+e_{(34)}-e_{(13)}-e_{(24)}-e_{(124)}-e_{(132)}-e_{(143)}-e_{(234)}+e_{(1324)} \\
& +e_{(1423)}-e_{(1234)}-e_{(1432)}+e_{(12)(34)}+e_{(13)(24)}+e_{(14)(23)}
\end{array}
$$

y por lo tanto

$$
\begin{aligned}
c_{(2,2)}= & e_{i d}+e_{(12)}+e_{(34)}-e_{(13)}-e_{(24)}-e_{(124)}-e_{(132)}-e_{(143)}-e_{(234)}+e_{(1324)} \\
& +e_{(1423)}-e_{(1234)}-e_{(1432)}+e_{(12)(34)}+e_{(13)(24)}+e_{(14)(23)}
\end{aligned}
$$

es el simetrizador de Young correspondiente a la partición $\lambda=(2,2)$. Mediante un cálculo directo (aunque un tanto laborioso) se puede verificar que $c_{(2,2)}^{2}=12 c_{(2,2)}$. Luego, $n_{(2,2)} y$ $V_{(2,2)}=\left(\mathbb{C} S_{4}\right) \cdot c_{(2,2)}$ es una representación irreducible de $S_{4}$, según el teorema 3.26.

\subsection{La Construcción de Weyl}

En la subsección anterior se vió que para cualquier partición $\lambda \vdash n$ se tiene un simetrizador de Young $c_{\lambda}$ en $\mathbb{C} S_{n}$. Se denota la imagen de $c_{\lambda}$ sobre $V^{\otimes n}$ por $\mathbb{S}_{\lambda} V$ :

$$
\mathbb{S}_{\lambda} V:=\operatorname{Im}\left(c_{\lambda} \mid V^{\otimes n}\right)
$$

la cual es una representación de GL $(V)$ [4, pág. 76].

Definición 3.28. La asignación $V \rightsquigarrow \mathbb{S}_{\lambda} V$, es un funtor que va de la categoría de espacios vectoriales en sí misma, denominado funtor de Schur, módulo de Weyl o simplemente construcción de Weyl, correspondiente a $\lambda$ [4, pág. 76].

Para probar la funtorialidad de $\mathbb{S}_{\lambda} V$ se requiere del siguiente lema.

Lema 3.29. Sean $\psi: V \longrightarrow W y \varphi: W \longrightarrow Z$ transformaciones lineales. Entonces

$$
(\varphi \circ \psi)^{\otimes n}=\varphi^{\otimes n} \circ \psi^{\otimes n}
$$

Prueba. Es suficiente probarlo para los generadores. Sea pues $v_{1} \otimes \cdots \otimes v_{n} \in V^{\otimes n}$ un generador. Luego

$$
\begin{aligned}
(\varphi \circ \psi)^{\otimes n}\left(v_{1} \otimes \cdots \otimes v_{n}\right) & =(\varphi \circ \psi)\left(v_{1}\right) \otimes \cdots \otimes(\varphi \circ \psi)\left(v_{n}\right) \\
& =\varphi\left(\psi\left(v_{1}\right)\right) \otimes \cdots \otimes \varphi\left(\psi\left(v_{n}\right)\right) \\
& =\varphi^{\otimes n}\left(\psi\left(v_{1}\right) \otimes \cdots \otimes \psi\left(v_{n}\right)\right) \\
& =\varphi^{\otimes n}\left(\psi^{\otimes n}\left(v_{1} \otimes \cdots \otimes v_{n}\right)\right) \\
& =\left(\varphi^{\otimes n} \circ \psi^{\otimes n}\right)\left(v_{1} \otimes \cdots \otimes v_{n}\right) .
\end{aligned}
$$

Por lo tanto, $(\varphi \circ \psi)^{\otimes n}=\varphi^{\otimes n} \circ \psi^{\otimes n}$. 
Proposición 3.30 (Funtorialidad de $\mathbb{S}_{\lambda}$ ). Sea $\psi: V \longrightarrow W$ una transformación lineal. Entonces $\mathbb{S}_{\lambda}(\psi): \mathbb{S}_{\lambda} V \longrightarrow \mathbb{S}_{\lambda} W$ satisface lo siguiente:

(1) $\mathbb{S}_{\lambda}(\varphi \circ \psi)=\mathbb{S}_{\lambda}(\varphi) \circ \mathbb{S}_{\lambda}(\psi)$, para toda transformación lineal $\varphi: W \longrightarrow Z$.

(2) $\mathbb{S}_{\lambda}\left(i d_{V}\right)=i d_{\mathbb{S}_{\lambda} V}$

\section{Prueba.}

(1) Sea $\left(v_{1} \otimes \cdots \otimes v_{n}\right) \cdot c_{\lambda} \in \mathbb{S}_{\lambda} V$, entonces

$$
\begin{aligned}
\mathbb{S}_{\lambda}(\varphi \circ \psi)\left(\left(v_{1} \otimes \cdots \otimes v_{n}\right) \cdot c_{\lambda}\right) & =(\varphi \circ \psi)^{\otimes n}\left(\left(v_{1} \otimes \cdots \otimes v_{n}\right) \cdot c_{\lambda}\right) \\
\stackrel{(3.29)}{=} & \left(\varphi^{\otimes n} \circ \psi^{\otimes n}\right)\left(\left(v_{1} \otimes \cdots \otimes v_{n}\right) \cdot c_{\lambda}\right) \\
= & \varphi^{\otimes n}\left(\psi^{\otimes n}\left(\left(v_{1} \otimes \cdots \otimes v_{n}\right) \cdot c_{\lambda}\right)\right) \\
= & \varphi^{\otimes n}\left(\left(\psi\left(v_{1}\right) \otimes \cdots \otimes \psi\left(v_{n}\right)\right) \cdot c_{\lambda}\right) \\
= & \mathbb{S}_{\lambda}(\varphi)\left(\left(\psi\left(v_{1}\right) \otimes \cdots \otimes \psi\left(v_{n}\right)\right) \cdot c_{\lambda}\right) \\
= & \mathbb{S}_{\lambda}(\varphi)\left(\mathbb{S}_{\lambda}(\psi)\left(\left(v_{1} \otimes \cdots \otimes v_{n}\right) \cdot c_{\lambda}\right)\right) \\
= & \left(\mathbb{S}_{\lambda}(\varphi) \circ \mathbb{S}_{\lambda}(\psi)\right)\left(\left(v_{1} \otimes \cdots \otimes v_{n}\right) \cdot c_{\lambda}\right)
\end{aligned}
$$

de donde $\mathbb{S}_{\lambda}(\varphi \circ \psi)=\mathbb{S}_{\lambda}(\varphi) \circ \mathbb{S}_{\lambda}(\psi)$.

(2) Sea $\left(v_{1} \otimes \cdots \otimes v_{n}\right) \cdot c_{\lambda} \in \mathbb{S}_{\lambda} V$. Entonces:

$$
\begin{aligned}
\mathbb{S}_{\lambda}\left(\operatorname{id}_{V}\right)\left(\left(v_{1} \otimes \cdots \otimes v_{n}\right) \cdot c_{\lambda}\right) & =\operatorname{id}_{V}^{\otimes n}\left(\left(v_{1} \otimes \cdots \otimes v_{n}\right) \cdot c_{\lambda}\right) \\
& =\left(\operatorname{id}_{V}\left(v_{1}\right) \otimes \cdots \otimes \operatorname{id}_{V}\left(v_{n}\right)\right) \cdot c_{\lambda} \\
& =\left(v_{1} \otimes \cdots \otimes v_{n}\right) \cdot c_{\lambda} .
\end{aligned}
$$

Por lo tanto, $\mathbb{S}_{\lambda}\left(\mathrm{id}_{V}\right)=\mathrm{id}_{\mathbb{S}_{\lambda} V}$ y la proposición queda demostrada.

Para la partición $\lambda=(n)$, la representación $V_{(n)}$ tiene asociada el funtor de Schur $\mathbb{S}_{(n)} V=\operatorname{Sym}^{n} V$; en tanto que para la partición $\lambda=\left(1^{n}\right)$, el funtor de Schur asociado a la representación $V_{\left(1^{n}\right)}$ es $\mathbb{S}_{\left(1^{n}\right)} V=\bigwedge^{n} V$ (cfr. el ejemplo 3.25).

\section{La Teoría de Caracteres}

La teoría de caracteres, estrechamente relacionada con la teoría de representaciones de grupos finitos, fué definida formalmente y generalizada por el matemático alemán Heinrich Martin Weber en 1881. La definición de Weber era una abstracción de una dada tres años antes por Dedekind, quien a su vez se inspiró más o menos directamente de Gauss, por el uso implícito que hace éste de caracteres de orden dos en sus Disquisitiones Arithmeticae publicadas en 1801 [1, pág. 5]. Weber define un caracter de un grupo abeliano finito $G$ como un 
homomorfismo $g \mapsto \chi(g)$ de $G$ al grupo multiplicativo de los números complejos de módulo uno. Luego, el conjunto $\hat{G}$ de todos los caracteres de $G$ es también un grupo abeliano finito bajo la multiplicación. Además, cada función complejo-valuada $f$ sobre $G$ puede escribirse de forma única como una combinación lineal de caracteres [1, pág. 6].

Anteriormente se hizo la observación de que un grupo finito $G$ admite sólo un número finito de representaciones irreducibles $V_{i}$, únicas salvo isomorfismo. Aquí yace la importancia de los teoremas de clasificación en teoría de representaciones: una vez descritas las representaciones irreducibles de $G$, podremos describir una representación arbitraria como una suma directa de las primeras. En consecuencia, dos importantes objetivos de la teoría son:

1) Describir todas las representaciones irreducibles de $G$.

2) Encontrar técnicas para dar una descomposición en suma directa (véase 3.17), y determinar las multiplicidades $a_{i}$ de una representación arbitraria $V$.

En álgebra lineal es conocida la propiedad de que cualesquiera dos matrices asociadas a una transformación lineal tienen la misma traza, lo que da sentido al concepto que sigue.

Definición 4.1. La función $\chi_{V}$ del grupo $G$ al campo $\mathbb{C}$ de los números complejos dada por

$$
\begin{array}{rlc}
\chi_{V}: G & \longrightarrow & \mathbb{C} \\
g & \longmapsto \operatorname{Tr}[\rho(g)]
\end{array}
$$

se denomina caracter ${ }^{4}$ de la representación $(V, \rho)$.

Del ejemplo 3.4 se sigue que el caracter de la representación trivial de un grupo $G$ es uno.

Teorema 4.2 (Algunas propiedades del caracter). Sea $G$ un grupo y sea $\chi_{V}$ el caracter de una representación $(V, \rho)$ de grado $n$. Entonces

(1) La transformación lineal $\rho(g): V \longrightarrow V$ es diagonalizable, para todo $g \in G$.

(2) $\chi_{V}(1)=n$, donde el 1 denota el neutro del grupo.

(3) $\chi_{V}\left(h g h^{-1}\right)=\chi_{V}(g)$, para todo $g, h \in G$.

PruebA. Se probarán únicamente los numerales (2) y (3).

(1) Como $\rho(1)=\mathrm{id}_{V}$, entonces $[\rho(1)]=I_{n}$ y así $\chi(1)=\operatorname{Tr}\left(I_{n}\right)=n$, donde $I_{n}$ es la matriz identidad de tamaño $n \times n$.

\footnotetext{
${ }^{4}$ Más adelante se verá el porqué del término para nombrar la función $\chi_{V}$.
} 
(2) El cálculo es directo:

$$
\begin{aligned}
\chi_{V}\left(h g h^{-1}\right) & =\operatorname{Tr}\left(\rho\left(h g h^{-1}\right)\right) \\
& =\operatorname{Tr}\left(\rho(h) \rho(g) \rho\left(h^{-1}\right)\right) \\
& =\operatorname{Tr}\left(\rho(h) \rho(g) \rho(h)^{-1}\right) \\
& =\operatorname{Tr}\left(\rho(h)^{-1} \rho(h) \rho(g)\right) \\
& =\operatorname{Tr}\left(\operatorname{id}_{V} \rho(g)\right) \\
& =\operatorname{Tr}(\rho(g)) .
\end{aligned}
$$

Ejemplo 4.3. Sea $(V, \rho)$ la representación regular ${ }^{5}$ de grado $n$ de un grupo $G \neq\{1\}$ de orden también $n$, y sea $\chi$ su caracter (se omite el subindice). Si $\left\{v_{g}\right\}_{g \in G}$ es una base de $V$, por la definición de representación regular se tiene que $\rho(\sigma) v_{g}=v_{\sigma g}$. Observe que la igualdad $\rho(\sigma) v_{g}=v_{g}$ se cumple si y sólo si $\sigma g=g$, la cual se verifica si y sólo si $\sigma=1$. Luego, si $[\rho(\sigma)]$ es la matriz asociada a $\rho(\sigma)$ en la base regular, entonces:

(1) Si $\sigma \neq 1$ (el neutro de $G$ ), la diagonal de $[\rho(\sigma)]$ consiste de ceros.

(2) Si $\sigma=1$, la diagonal de $[\rho(1)]$ consiste de unos.

Por lo tanto, el caracter $\chi$ de la representación regular está dado por la igualdad

$$
\chi(\sigma)=\left\{\begin{array}{lll}
0 & \text { si } & \sigma \neq 1 \\
n & \text { si } & \sigma=1
\end{array}\right.
$$

Proposición 4.4. Sean $\left(V_{1}, \rho\right)$ y $\left(V_{2}, \tilde{\rho}\right)$ dos representaciones de un grupo $G$, de grados $m$ y $n$ respectivamente, con caracteres $\chi_{V_{1}} y \chi_{V_{2}}$. Entonces:
(1) $\chi_{V_{1} \oplus V_{2}}=\chi_{V_{1}}+\chi_{V_{2}}$.
(2) $\chi_{V_{1} \otimes V_{2}}=\chi_{V_{1}} \cdot \chi_{V_{2}}$.

Prueba.

(1) Sean $V=V_{1} \oplus V_{2}$ y $\mathcal{B}_{1}, \mathcal{B}_{2}$ bases de $V_{1}$ y $V_{2}$ respectivamente; entonces $\mathcal{B}=\mathcal{B}_{1} \cup \mathcal{B}_{2}$ es una base de $V$ y la matriz asociada a $(\rho \oplus \tilde{\rho})(g)$ es de la forma (cfr. con (1) de 4.2)

$$
[\rho \oplus \widetilde{\rho}]_{\mathcal{B}}=\left(\begin{array}{cc}
{[\rho(g)]_{\mathcal{B}_{1}}} & 0 \\
0 & {[\tilde{\rho}(g)]_{\mathcal{B}_{2}}}
\end{array}\right)
$$

de donde

$$
\chi_{V_{1} \oplus V_{2}}(g)=\operatorname{Tr}[(\rho \oplus \tilde{\rho})(g)]_{\mathcal{B}}=\operatorname{Tr}[\rho(g)]_{\mathcal{B}_{1}}+\operatorname{Tr}[\tilde{\rho}(g)]_{\mathcal{B}_{2}}=\chi_{V_{1}}(g)+\chi_{V_{2}}(g) .
$$

\footnotetext{
${ }^{5}$ Véase el ejemplo 3.6 .
} 
(2) Si $v_{j}$ y $w_{k}$ son vectores propios asociados a $\lambda_{j}$ y $\mu_{k}$ respectivamente, entonces

$$
(\rho(g) \otimes \tilde{\rho}(g))\left(v_{j} \otimes w_{k}\right)=\rho(g)\left(v_{j}\right) \otimes \tilde{\rho}(g)\left(w_{k}\right)=\lambda_{j} v_{j} \otimes \mu_{k} w_{k}=\left(\lambda_{j} \mu_{k}\right)\left(v_{j} \otimes w_{k}\right)
$$

lo cual implica que $\lambda_{j} \mu_{k}$ es valor propio de $v_{j} \otimes w_{j}$. Esto es, $\left\{v_{j} \otimes w_{j}\right\}_{j=1, k=1}^{m, n}$ es una base de vectores propios de $\rho(g) \otimes \tilde{\rho}(g)$. Luego

$$
\begin{aligned}
\chi_{V_{1} \otimes V_{2}} & =\operatorname{Tr}[\rho(g) \otimes \tilde{\rho}(g)] \\
& =\sum_{j=1}^{m} \sum_{k=1}^{n} \lambda_{j} \mu_{k} \\
& =\left(\sum_{j=1}^{m} \lambda_{j}\right)\left(\sum_{k=1}^{n} \mu_{k}\right) \\
& =\operatorname{Tr}[\rho(g)] \cdot \operatorname{Tr}[\tilde{\rho}(g)] \\
& =\chi_{V_{1}} \cdot \chi_{V_{2}}
\end{aligned}
$$

Definición 4.5. Sean $\chi$ y $\psi$ los caracteres de dos representaciones de un grupo G. Como en este trabajo sólo se están considerando grupos finitos, la suma

$$
\langle\chi, \psi\rangle:=\frac{1}{|G|} \sum_{g \in G} \chi(g) \psi\left(g^{-1}\right)
$$

es finita y se comporta como un producto interno, en el sentido de que es bilineal, simétrica $y\langle\chi, \chi\rangle \neq 0$ para todo caracter $\chi$.

El resultado que sigue permite determinar ciertas características de las representaciones a través de la ortogonalidad de sus caracteres.

Teorema 4.6. [8, pág. 189] Sean $V$ y $W$ representaciones de un grupo $G$. Luego

(1) Si $V$ y $W$ son irreducibles no isomorfas, entonces sus caracteres son ortogonales, i.e. $\left\langle\chi_{V}, \chi_{W}\right\rangle=0$.

(2) Si $V$ es irreducible, entonces $\left\langle\chi_{V}, \chi_{V}\right\rangle=1$.

Nótese que el primer inciso da una condición necesaria para la irreducibilidad de una representación. Por su parte, el segundo inciso nos da una condición necesaria para la irreducibilidad y no isomorfía de dos representaciones.

Ejemplo 4.7. Sea $(V, \rho)$ la representación regular ${ }^{6}$ de grado $n$ de un grupo $G \neq\{1\}$ de orden

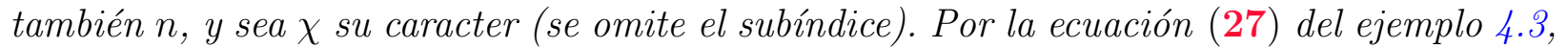
se tiene que $\chi(g)=0$ si $g \neq 1, y \chi(1)=n$, de donde

$$
\langle\chi, \chi\rangle \stackrel{(28)}{=} \frac{1}{n} \sum_{g \in G} \chi(g) \chi\left(g^{-1}\right)=\frac{1}{n} \chi(1) \chi(1)=\frac{1}{n} n \cdot n=n \neq 1 .
$$

Por consiguiente, la representación regular de grado $n>1$ no es irreducible.

\footnotetext{
${ }^{6}$ Véase el ejemplo 3.6.
} 
Teorema 4.8. Sean $(V, \rho)$ y $(W, \tilde{\rho})$ dos representaciones de un grupo $G$. Entonces

(a) Si $V$ admite una descomposición en representaciones irreducibles distintas

$$
V=V_{1}^{\oplus a_{1}} \oplus \cdots \oplus V_{k}^{\oplus a_{k}}
$$

entonces $\left\langle\chi_{V}, \chi_{V_{i}}\right\rangle=a_{i}$. Además, $a_{i}$ es independiente de la descomposición elegida.

(b) $V \cong W$ si y sólo si $\chi_{V}=\chi_{W}$.

(c) $S i\left\langle\chi_{V}, \chi_{V}\right\rangle=1$, entonces $V$ es irreducible.

\section{PRUEBA.}

(a) Se sigue del numeral (1) de la proposición 4.4 y del teorema 4.6, ya que

$$
\chi_{V}=\chi_{V_{1}^{\oplus a_{1} \oplus \cdots \oplus V_{k}^{\oplus a k}}}=\chi_{V_{1}^{\oplus a_{1}}}+\cdots+\chi_{V_{1}^{\oplus a_{k}}}=a_{1} \chi_{V_{1}}+\cdots+a_{k} \chi_{V_{k}}
$$

$(\boldsymbol{b}) \Longrightarrow)$ Supongamos que $V \cong W$ y sea $\phi: V \longrightarrow W$ un isomorfismo. Entonces para toda $g \in G$ se tiene que $\phi \circ \rho(g)=\tilde{\rho}(g) \circ \phi$, de donde $\rho(g)=\phi^{-1} \circ \tilde{\rho}(g) \circ \phi$, y por lo tanto $\chi_{V}(g)=\operatorname{Tr}(\rho(g))=\operatorname{Tr}\left(\phi^{-1} \circ \tilde{\rho}(g) \circ \phi\right)=\operatorname{Tr}(\tilde{\rho}(g))=\chi_{W}(g)$.

$\Longleftarrow)$ Supongamos ahora que $\chi_{V}=\chi_{W}$. Por el inciso $(a), \rho(g)$ y $\tilde{\rho}(g)$ contienen, cada uno, una representación irreducible el mismo número de veces. Por lo tanto, $V \cong W$.

(c) Supongamos que $\left\langle\chi_{V}, \chi_{V}\right\rangle=1$ y sea $V_{1}^{\oplus a_{1}} \oplus \cdots \oplus V_{k}^{\oplus a_{k}}$ la descomposición en irreducibles de $V$. Luego

$$
\begin{aligned}
\left\langle\chi_{V}, \chi_{V}\right\rangle & =\left\langle\chi_{V}, a_{1} \chi_{V_{1}}+\cdots a_{k} \chi_{V_{k}}\right\rangle \\
& =a_{1}\left\langle\chi_{V}, \chi_{V_{1}}\right\rangle+\cdots+a_{k}\left\langle\chi_{V}, \chi_{V_{k}}\right\rangle \\
& \stackrel{(a)}{=} a_{1} \cdot a_{1}+\cdots+a_{k} \cdot a_{k} \\
& =\sum_{i=1}^{k} a_{i}^{2} \\
& =1 .
\end{aligned}
$$

De modo que algún $a_{i}$ es 1 y todos los demás son cero, de donde $V \cong V_{i}$ y por lo tanto $V$ es irreducible.

Nótese que mientras la proposición 3.17 garantiza la existencia de una descomposición en irreducibles de una representación $V$, el teorema 4.8 en su inciso (a) permite calcular los $a_{i}$ con independencia de la descomposición elegida; el inciso (b) es el porqué del término caracter al que hace referencia el pie de página de la definición 4.1: la función $\chi_{V}$ caracteriza a la representación $(V, \rho)$ en el sentido de que dos representaciones con el mismo caracter son isomorfas; y finalmente, en su inciso (c) proporciona una condición suficiente para la irreducibilidad de una representación. Esto prueba el poder de la teoría de caracteres. 


\section{Conclusiones}

Si bien los resultados discutidos no son todos los que se conocen, estos dan un fundamento sólido a la teoría de representaciones de grupos finitos y proporcionan una razón para profundizar más en ella, así como también en la teoría de caracteres. Aun cuando su alcance no se limita sólo al ámbito matemático, se optó por dar un panorama breve pero lo más general posible dentro del mismo, con el fin de que el lector pueda apreciar la belleza de las matemáticas detrás de estas poderosas herramientas. Para lograr también dicho objetivo, se incluyó una gran cantidad de ejemplos, expuestos (en su mayoría) con gran detalle, haciendo más amenas las ideas y los conceptos presentados aquí.

Los autores esperan que este trabajo sirva de motivación a la comunidad científica para continuar con el estudio de la teoría de representaciones, sus alcances y aplicaciones en otras ramas de la ciencia además de las matemáticas, la física y la química.

\section{Referencias}

[1] Atiyah, M.F. Et Al. Representation Theory of Lie Groups, Cambridge University Press (London Mathematical Society Lecture Note Series 34), Cambridge (1980).

[2] Cordero, Patricio. Grupos de simetría en mecánica cuántica, Departamento de Física, Facultad de Ciencias Físicas y Matemáticas, Universidad de Chile (2007), https://www.cec.uchile.cl/cinetica/pcordero/todos/GruposEnQM.pdf.

[3] Etingof, Pavel. 18.712 Introduction to Representation Theory. Fall 2010. Massachusetts Institute of Technology: MIT: OpenCourseWare, http://ocw.mit.edu. License: Creative Commons BY-NC-SA.

[4] Fulton, W. and Harris, J. Representation Theory. A First Course, Springer-Verlag (Graduate Texts in Mathematics), USA (2004).

[5] Hardy, G.H. and Ramanujan, S. Asymptotic Formula in Combinatory Analysis, Proceedings of the London Mathematical Society s2-17, 75-115 (1918). DOI: $10.1112 / \mathrm{plms} / \mathrm{s} 2-17.1 .75$

[6] LAm, T.Y. Representations of Finite Groups: A Hundred Years, Part I, Notices of the AMS 45, 361-372 (1998).

[7] Poveda Pinilla, Arnulfo and Perilla de Zambrano, Isabel Cristina. Curso de Simetría en Química, Departamento de Química, Facultad de Ciencias, Universidad Nacional de Colombia - Sede Bogotá, http://168.176.239.58/cursos/ciencias/ 2000189_2/index.html. License: Creative Commons BY-NC-ND 2.5 CO.

[8] Zaldívar, FeliPe. Introducción a la Teoría de Grupos, Sociedad Matemática Mexicana (Aportaciones Matemáticas 34), México (2009). 